\title{
Ponderomotive impacts of ion cyclotron waves on the ions in the equatorial zone of the magnetosphere
}

\author{
A. Guglielmi ${ }^{1}$, K. Hayashi ${ }^{2}$, R. Lundin ${ }^{3}$, and ${ }^{4}$ A. Potapov \\ ${ }^{1}$ United Institute of Physics of the Earth, Moscow, Russia \\ ${ }^{2}$ University of Tokyo, Tokyo, Japan \\ ${ }^{3}$ Swedish Institute of Space Physics, Kiruna, Sweden \\ ${ }^{4}$ Institute of Solar-Terrestrial Physics, Irkutsk, Russia
}

(Received October 12, 1998; Revised April 12, 1999; Accepted May 7, 1999)

\begin{abstract}
The ponderomotive influence of ion cyclotron waves on the field-aligned distribution and motion of ions in the equatorial zone of the magnetosphere is examined. The hydrodynamic, quasi-hydrodynamic and "test-particle" approaches are used for the study of ponderomotive wave-particle interaction. Particular attention has been given to the challenging questions encountered in applying the general theory to the magnetospheric physics. The closed system of quasi-linear equations describing the ponderomotive effects is derived. Analytical investigation of the basic equations has demonstrated the diverse manifestations of the ponderomotive impact of ion cyclotron waves on the ion population in the magnetosphere. It is found that the redistribution of ion density under the action of ponderomotive force with increase in the wave amplitude follows the pattern of phase transition of the second kind. The density distribution changes qualitatively as the point in plane of the governing parameters of system crosses a demarcation line. It has been found that the magnetic equator is an attractor for heavy ion. The period of the finite (oscillatory) motion of a heavy ion, which is trapped in the potential trough in the vicinity of magnetic equator, depends on the wave frequency, wave amplitude, together with the energy of motion. In addition, the diffusion equilibrium of ions in a multicomponent plasma is considered, and the ponderomotive separation of ions in a binary mixture is demonstrated. It is shown that the heavy ions collect near the magnetic equator provided the waves are comparatively strong. It suggests that the ponderomotive effects play a part in formation of structure and dynamics of the magnetosphere.
\end{abstract}

\section{Introduction}

Ponderomotive force is the time-averaged nonlinear force acting on the media in the presence of high frequency electromagnetic field. Nonlinearity is herein taken to mean that the ponderomotive force is quadratic in the amplitude of oscillations. The conventional term "high frequency" should be read in the context of presented paper as denoting the frequency range of electromagnetic ion cyclotron waves in the Earth's magnetosphere, i.e., approximately $0.1-10 \mathrm{~Hz}$ in compliance with the numerous satellite observations (e.g., Bossen et al., 1976; Perraut et al., 1984; Lundin and Hultqvist, 1989; Anderson et al., 1992a,b; Erlandson et al., 1990, 1992; Gustafsson et al., 1990; Lundin et al., 1990; Fraser et al., 1992; LaBelle and Treumann, 1992; Mursula et al., 1994; Anderson, 1996). It suggests that the ponderomotive effects play a part in formation of structure and dynamics of the magnetosphere (Lundin, 1988; Lundin and Hultqvist, 1989; Allan et al., 1991; Lundin and Eliasson, 1991; Allan, 1992, 1993a,b; Guglielmi, 1992, 1997; Guglielmi et al., 1995, 1996; Hultqvist, 1996; Kangas et al., 1998). However, till recently the theory of ponderomotive wave-particle interaction had been played a moderate role in determination

Copy right (c) The Society of Geomagnetism and Earth, Planetary and Space Sciences (SGEPSS); The Seismological Society of Japan; The Volcanological Society of Japan; The Geodetic Society of Japan; The Japanese Society for Planetary Sciences. of the intimate relations between the experimental findings. This is in part because of the severity of the concept of averaged forces acting on the plasma immersed into a high frequency electromagnetic field.

A note of historical interest is appropriate here. The hypothesis on the light pressure (this is one of a number of ponderomotive effects) goes back to J. Kepler. Electromagnetic theory of the light pressure was first proposed by J. Maxwell in 1873. It was one of the prominent results of classical electrodynamics. P. Lebedev was the first to measure the light pressure in 1899. Early in the century M. Abraham and G. Minkowski launched an in-depth analysis of problem, based on the concept of electromagnetic field momentum in the framework of macroscopic electrodynamics (see, for example, Ginzburg, 1975). In the modern time, Pitayevsky (1960), Washimi and Karpman (1976) pursued this line of inquire.

Here the following comment is in order. Except for the gravity force, the Lorentz force

$$
\mathbf{F}=e \mathbf{E}+\frac{1}{c} \mathbf{v} \times \mathbf{B}
$$

is the unique fundamental force acting on the magnetospheric particles. Here $\mathbf{E}$ and $\mathbf{B}$ are the electric and magnetic fields, $e$ is the electric charge, $\mathbf{v}$ is the velocity of particle, and $c$ is the velocity of light. In this respect the ponderomotive force is an auxiliary notion. It may be introduced phenomenologically 
as mentioned above, but other than this, it may be found by averaging $\mathbf{F}$ over the time in the framework of a microscopic model of the medium. Miller(1958) had presented the known expression for the force which is proportional to $\nabla\left\langle E^{2}\right\rangle$ where the angular parenthesis denotes the averaging over the time. Hence, the Miller force is related to the spatial inhomogeneity of the wave field. Lundin and Hultqvist (1989) proposed the so-called "magnetic moment pumping" which is proportional to $\left\langle E^{2}\right\rangle \nabla_{\|} \ln B$ where $\nabla_{\|}$is the spatial derivative along the lines of geomagnetic field B. Beyond question that both of these components of ponderomotive force are operating in the magnetosphere. However, it is appropriate at this point to note that the Lundin-Hultqvist force is paramount for the ion cyclotron waves, whereas the Miller force prevails for the Alfvén waves.

In spite of the fact that a considerable body of work devoted to ponderomotive wave-particle interaction has already been published in geophysical literature, much remains to be done in this area. Our point is that we have still a long way to go toward the understanding the most interesting manifestations of ponderomotive forces. The purpose of the presented work is to examine the ponderomotive influence of the travelling ion cyclotron waves on the field-aligned distribution and motion of ions in the equatorial zone of magnetosphere. The work is not intended to be an exhaustive treatise of the subject. The main attention has been given to the challenging questions encountered in applying the general theory to the magnetospheric physics.

In Section 2 the basic equations are described. The closed system of equations of the 1D steady state quasi-hydrodynamics is presented with allowance made for the ponderomotive forces. In Section 3 it is shown that the problem on the ponderomotive impact of the waves upon the plasma density may be formally reduced into a problem on phase transition of the second kind. In such a formalism, the plasma distribution is altered radically as the imaging point in plane of the governing parameters crosses some demarcation line. In Sections 4 and 5 we seek the approaches to the problem of ponderomotive interaction of the heavy ions with ion cyclotron waves in the equatorial zone of magnetosphere. Here we come up against the problem of self-consistency of the wave field, and the problem of singularity appearing in the expression of the ponderomotive force at the gyrofrequency of heavy ions. The brief discussion in Section 6 touches both the prospects for uses of the concept of ponderomotive force in the magnetospheric physics, and the obstacles interposed on this way.

\section{Basic Equations}

\subsection{Hydrodynamics}

The concept of ponderomotive forces may be brought into the wave theory phenomenologically (Landau and Lifshitz, 1984). A rather general phenomenological expressions were obtained by Pitayevsky (1960), Washimi and Karpman (1976). For reference we have presented here the corresponding formulae. For the sake of convenience the force $\mathbf{f}=\mathbf{f}^{(1)}+\mathbf{f}^{(2)}$ acting on the unit volume is divided into the time-dependent component

$$
\begin{aligned}
\mathbf{f}^{(1)}= & \frac{1}{8 \pi c} \operatorname{Re}\left\{\frac{\partial}{\partial t}\left[(\mathbf{D}-\mathbf{E}) \times \mathbf{b}^{*}\right]+\omega\left(\mathbf{K} \times \mathbf{b}^{*}\right)\right\} \\
& +\frac{1}{16 \pi} \operatorname{Im} E_{\alpha}^{*} \frac{\partial E_{\beta}}{\partial t} \frac{\partial^{2} \epsilon_{\alpha \beta}}{\partial B_{\gamma} \partial \omega} \nabla B_{\gamma},
\end{aligned}
$$

where

$$
D_{\alpha}=\varepsilon_{\alpha \beta} E_{\beta}, K_{\alpha}=\frac{\partial \epsilon_{\alpha \beta}}{\partial \omega} \frac{\partial E_{\beta}}{\partial t},
$$

and time-independent component

$$
\begin{aligned}
\mathbf{f}^{(2)}= & \frac{1}{16 \pi}\left\{\left(\varepsilon_{\alpha \beta}-\delta_{\alpha \beta}\right) \nabla E_{\alpha}^{*} E_{\beta}\right. \\
& +E_{\alpha}^{*} E_{\beta} \frac{\partial \varepsilon_{\alpha \beta}}{\partial B_{\gamma}} \nabla B_{\gamma} \\
& \left.\cdot\left[\mathbf{B} \times \operatorname{curl}\left(\frac{\partial \epsilon_{\alpha \beta}}{\partial \mathbf{B}} E_{\alpha}^{*} E_{\beta}\right)\right]\right\} .
\end{aligned}
$$

Here $\mathbf{B}(\mathbf{x})$ is the external magnetic field, $\varepsilon_{\alpha \beta}(\omega, \mathbf{x})$ is the dielectric permeability, $\omega$ is the carrier frequency of the wave, $\delta_{\alpha \beta}$ is the Kroneker delta symbol. It is supposed that the electric field of the quasi-monochromatic wave have the form $\mathbf{E}(\omega, t ; \mathbf{x}) \exp (-i \omega t)$. The amplitude $\mathbf{E}(\omega, t ; \mathbf{x})$ varies in time slower than $\exp (-i \omega t)$, but the spatial distribution of the amplitude may be arbitrary. The same is also true for the amplitude $\mathbf{b}(\omega, t ; \mathbf{x})$ of the magnetic field oscillations (Pitayevsky, 1960; Washimi and Karpman, 1976).

On the one hand, the equations (1) and (2) are too cumbersome; on the other hand, as we shall see subsequently, these equations are not entirely adequate for our purposes. It is desirable to have the equations that are somewhat less intricate, and somewhat more general. To avoid confusion, it should be noted that the simplification and generalization refer to the distinct parts of the model, namely, to the spacetime structure of the wave field and to the ionic composition of the medium.

So, let us impose the following restrictions on the wave field. Firstly, we shall avoid the difficulties connected with the time-dependent component (1) of ponderomotive force by assuming that the characteristic time of the wave amplitude modulation is far in excess of the travel time during which a wave packet travels a distance of the order of characteristic length of the wave field. Then the longitudinal component of the ponderomotive force is

$$
\begin{aligned}
\mathbf{f}_{\|}= & \frac{1}{16 \pi}\left[\left(\varepsilon_{\alpha \beta}-\delta_{\alpha \beta}\right) \nabla_{\|} E_{\alpha}^{*} E_{\beta}\right. \\
& \left.+E_{\alpha}^{*} E_{\beta} \frac{\partial \varepsilon_{\alpha \beta}}{\partial B_{\gamma}} \nabla_{\|} B_{\gamma}\right],
\end{aligned}
$$

where $\nabla_{\|}$is the spatial derivative along a field line of $\mathbf{B}$. Hereinafter, we shall consider the ponderomotive redistribution of ions along the geomagnetic field lines, so that the transverse component of the ponderomotive force $\mathbf{f}_{\perp}=\mathbf{f}-\mathbf{f}_{\|}$ is of no interest. (It can be shown that the influence of $\mathbf{f}_{\perp}$ is negligible as long as $c_{S}^{2} \ll c_{A}^{2}$ where $c_{S}$ is the sound velocity, $c_{A}$ is the Alfvén velocity.) 
Secondly, we shall consider the circularly polarized ion cyclotron waves. Let us introduce the local Cartesian coordinate system $(x, y, z)$ so that the external magnetic field $\mathbf{B}=(0,0, B)$. The electric field components of the wave are related by the law $E_{x}=i E_{y}$. This is expressible in the following manner: $\mathbf{E}=\mathbf{a} E_{x}$. Here $\mathbf{a}=(1,-i, 0)$ is the polarization vector. Now, we make use of the relation $\varepsilon_{\alpha \beta} a_{\alpha}^{*} a_{\beta}=$ $n^{2}$, where $n$ is the refractive index (see, e.g., Guglielmi and Pokhotelov, 1996). With this relation, equation (3) becomes

$$
f_{\|}=\frac{1}{8 \pi}\left[\frac{\partial n^{2}}{\partial B} E^{2} \nabla_{\|} B+\left(n^{2}-1\right) \nabla_{\|} E^{2}\right],
$$

where $n^{2}=\epsilon_{x x}-i \epsilon_{x y}, E=\left|E_{x}\right|$. Thirdly, we shall restrict our consideration to the travelling waves. Then

$$
\nabla_{\|} E^{2}=E^{2} \nabla_{\|} \ln (B / n)
$$

for the relatively short waves when the WKB approximation can be used. The equation (5) provides an approximate solution of the problem of self-consistency between the spatial structure of the wave field and the spatial distribution of the charged particles (Guglielmi, 1992). We will revert to the problem of self-consistency at the end of this subsection. Substitution of (5) into (4) gives:

$$
\begin{aligned}
f_{\|}= & \frac{E^{2}}{16 \pi}\left\{\left[\left(1+\frac{1}{n^{2}}\right) \frac{\partial n^{2}}{\partial B}\right.\right. \\
& \left.+\left(1-\frac{1}{n^{2}}\right) \frac{2 n^{2}}{B}\right] \nabla_{\|} B \\
& \left.-\left(1-\frac{1}{n^{2}}\right) \frac{\partial n^{2}}{\partial N} \nabla_{\|} N\right\} .
\end{aligned}
$$

Here $N$ is the number of electrons in the unit volume.

Now that we have presented the ponderomotive force, we need to focus upon the equation of motion. The force (6), it will be remembered, acts on the unit volume of the medium under the steady state conditions. Hence the hydrodynamic equation of motion is

$$
\rho u \nabla_{\|} u=-\nabla_{\|} p+\rho g_{\|}+f_{\|},
$$

and the mass conservation equation is

$$
\rho u / B=\text { const . }
$$

Here $u$ is the bulk velocity along the geomagnetic field lines, $\rho$ is the plasma density, $p$ is the plasma pressure, and $g_{\|}$ is the field aligned component of gravitation acceleration. These equations must be supplemented with the equation of state, for example, with the equation $p=c_{s}^{2} \rho$ in the event of isothermal motion. Lastly, the expression for $n^{2}$ is required. By using the known expression for $\varepsilon_{\alpha \beta}$ in the cold-plasma approximation we obtain

$$
n^{2}=1-\sum_{\mu} \frac{\omega_{0 \mu}^{2}}{\omega\left(\omega-\Omega_{\mu}\right)}
$$

where $\omega_{0 \mu}=\left(4 \pi e_{\mu}^{2} N_{\mu} / m_{\mu}\right)^{1 / 2}$ is the plasma frequency, $\Omega_{\mu}=e_{\mu} B / m_{\mu} c$ is the gyrofrequency, $e_{\mu}$ is the charge, $m_{\mu}$ is the mass, $N_{\mu}$ is the concentration of the particles of given type, the multi-index $\mu=(e, i)$ signifies the electrons $e$ and ions $i$, the summation is made over the particle species (see e.g., Ginzburg, 1971). The equation (9) may be used upon condition that $u \ll c / n$.

We can see that the spatial distribution of $n^{2}$ depends on the spatial distributions of the particle species. Let us suppose that the plasma consists of electrons and singly charged ions which are identical in mass. Then the quasi-neutrality condition

$$
\sum_{\mu} e_{\mu} N_{\mu}=0
$$

leads to the equations $N_{i}=N$ and $\rho=N m_{i}$. The square of refractive index (9) equals

$$
n^{2}=\left(\frac{c}{c_{A}}\right)^{2}\left(\frac{\Omega_{i}}{\Omega_{i}-\omega}\right),
$$

where $c_{A}=B /(4 \pi \rho)^{1 / 2}, \omega<\Omega_{i}, c_{A}^{2} \ll c^{2}$. The ponderomotive force (6) becomes

$$
f_{\|}=-\frac{b^{2}}{16 \pi}\left[\left(\frac{\omega}{\Omega_{i}-\omega}\right) \nabla_{\|} \ln B+\nabla_{\|} \ln \rho\right],
$$

and the self-consistency condition (5) becomes

$$
\frac{b^{2}}{\sqrt{\rho}}\left(1-\frac{\omega}{\Omega_{i}}\right)^{1 / 2}=\text { const . }
$$

Here $b=n E$ is the amplitude of the wave magnetic field.

The equations (7), (8), (12), (13) and the equation of state $p=c_{s}^{2} \rho$ make the closed system of equations. It is pertinent to note that the hydrodynamic model of plasma with isotropic pressure, as applied to the magnetosphere, is a palliative of a sort. In particular, this model fails to account the mirror force acting on the transversely heating ions (e.g., Singh, 1994; Barghouthi, 1997). A more general approach based on application of kinetic equations allows one to describe in detail the magnetospheric plasma. However, the application of the methods of physical kinetics is difficult mathematically. There are another challenging problems. Without going into details, we call attention to the fact that the abandonment of the hydrodynamic equations such as (7) leads to the conceptual difficulty, since the fundamental equations for the averaged (ponderomotive) forces are limited in use to the phenomenological methods of description of the media. The concept of average forces fails as soon as we should look at the method of kinetic equation for clue.

In conclusion of this subsection, we return to the very important equations (4) and (5). The equation (4) was deduced for the ion cyclotron waves in which the vector $\mathbf{E}$ has a counter-clockwise rotation in the plane, perpendicular to the direction of the B. However, it turns out that the equation (4) can also be applied to the waves with clockwise polarization. In that case the polarization vector and refractive index are equal to $\mathbf{a}=(1, i, 0)$, and $n^{2}=\epsilon_{x x}+i \epsilon_{x y}$ respectively. Hence, with the relation $\varepsilon_{\alpha \beta} a_{\alpha}^{*} a_{\beta}=n^{2}$ we can derive the equation (4) from Eq. (3) for the waves with the clockwise polarization. The equation (5) rests on the assumption that the ion cyclotron waves travel in a waveguide stretched along the geomagnetic field lines. Considering that the effective 
cross-section of the waveguide changes along the field lines as $B^{-1}$, the equation (5) can be obtained in the paraxial approximation (Guglielmi, 1992). It is significant that equation (5) furnish an opportunity to solve analytically some special problems of the ponderomotive wave-particle interaction. It is needless to say that there is a rich variety of the wave patterns, which defy analytical description. In general case the computer simulation is used for the study of the ponderomotive forces in the magnetosphere (e.g., Allan et al., 1991; Allan, 1992, 1993a,b).

The equation (5) provides a way of solving the problem of self-consistency between the spatial structure of the wave field and the spatial distribution of the particles. In general, account must be taken of the spatial as well as the temporal manifestations of the ponderomotive self action. This is a challenging task. In particular, this more rigorous treatment requires the consideration of the time-dependent component (1) of the ponderomotive force. So, for the sake of simplicity we are considering the amplitude of electromagnetic oscillations to be time independent. However, it is felt that our theory applies also to the slowly modulated oscillations provided that we deal with a steady-state modulation. In that case the value $E$ has a meaning of the electric field amplitude averaged over period of modulation.

\subsection{Quasi-hydrodynamics}

A completely different type of situation occurs in the case of multicomponent plasma. Here we run into obstacle in an attempt to close the system of equations. Really, in place of (7), (8), the system of quasi-hydrodynamic equations

$$
\begin{aligned}
& u_{i} \nabla_{\|} u_{i}=-\left(T / m_{i}\right) \nabla_{\|} \ln N_{i} \\
&+\left(e / m_{i}\right) E_{\|}+g_{\|}+A_{i}, \\
& \nabla_{\|} B^{-1} N_{i} u_{i}=0
\end{aligned}
$$

may be used; however, the expressions for the ponderomotive accelerations $A_{i}$ of ion species are unknown. Here $T$ is the temperature, $E_{\|}$is the ambipolar electric field, the index $i=1,2, \ldots$ labels the kinds of ions. It is assumed that the plasma is isothermal and each ion has the single electric charge $e$. The plasma density equals $\rho=\sum m_{i} N_{i}$. The quasi-neutrality equation (10) becomes

$$
\sum N_{i}=N
$$

where the summation is made over the ion species.

Guglielmi and Lundin (1999) have used a simple testparticle approach in the evaluation of the ponderomotive acceleration. Here we make an effort to evaluate $A_{i}$ by a rearrangement of terms in the phenomenological equations (1), (2).

First we write the total force as a sum of partial forces:

$$
\mathbf{f}=\sum_{\mu} \mathbf{f}_{\mu}
$$

Now, let us note that the tensor of dielectric permeability appears in the expression for the ponderomotive force $\mathbf{f}$ as follows: $\varepsilon_{\alpha \beta}-\delta_{\alpha \beta}$ (see Eqs. (1) and (2)). This combination equals

$$
\varepsilon_{\alpha \beta}-\delta_{\alpha \beta}=\frac{4 \pi i}{\omega} \sum_{\mu} \sigma_{\alpha \beta}^{\mu},
$$

where $\sigma_{\alpha \beta}^{\mu}$ is the contribution from particles $\mu$ to the tensor of conductivity. These considerations give us the rule to split the total force $\mathbf{f}$ into the sum of partial forces $\mathbf{f}_{\mu}$. Then we can to define the partial accelerations $\mathbf{A}_{\mu}=\rho_{\mu}^{-1} \mathbf{f}_{\mu}$, where $\rho_{\mu}=N_{\mu} m_{\mu}$, in particular,

$$
\begin{aligned}
A_{i} & =\frac{e^{2} E^{2}}{2 m_{i}^{2} \omega\left(\Omega_{i}-\omega\right)} \\
& \cdot\left[\nabla_{\|} \ln E^{2}-\left(\frac{\Omega_{i}}{\Omega_{i}-\omega}\right) \nabla_{\|} \ln B\right] .
\end{aligned}
$$

There is reason to hope that the expression (17) is true, since it is coincident with the expression which was obtained by the another method (Guglielmi and Lundin, 1999).

By using the self-consistency condition (5), we can rewrite (17) in the form

$$
\begin{aligned}
A_{i}= & -\frac{e^{2} E^{2}}{2 m_{i}^{2} \omega\left(\Omega_{i}-\omega\right)} \\
& \cdot\left[\left(\frac{\omega}{\Omega_{i}-\omega}\right) \nabla_{\|} \ln B+\frac{1}{2} \nabla_{\|} \ln n^{2}\right] .
\end{aligned}
$$

We would like to exclude the derivative of $n^{2}$ by using the relation

$$
\nabla_{\|} n^{2}=\frac{\partial n^{2}}{\partial B} \nabla_{\|} B+\sum_{\mu} \frac{\partial n^{2}}{\partial N_{\mu}} \nabla_{\|} N_{\mu}
$$

It is conveniently to present (9) as follows:

$$
\begin{aligned}
& n^{2}=1+\sum_{\mu}\left(n_{\mu}^{2}-1\right), \\
& n_{\mu}^{2}=1-\omega_{0 \mu}^{2} / \omega\left(\omega-\Omega_{\mu}\right) .
\end{aligned}
$$

Then

$$
\begin{aligned}
& \frac{\partial n^{2}}{\partial \ln B}=\sum_{\mu} \frac{\partial n_{\mu}^{2}}{\partial \ln B}=\sum_{\mu}\left(\frac{\Omega_{\mu}}{\omega-\Omega_{\mu}}\right)\left(n_{\mu}^{2}-1\right), \\
& \frac{\partial n^{2}}{\partial \ln N_{\mu}}=n_{\mu}^{2}-1,
\end{aligned}
$$

and the net result is

$$
\begin{aligned}
A_{i}= & -\frac{e^{2} E^{2}}{2 m_{i} \omega\left(\Omega_{i}-\omega\right)}\left\{\left(\frac{\omega}{\Omega_{i}-\omega}\right) \nabla_{\|} \ln B\right. \\
& +\frac{1}{2 n^{2}} \sum_{\mu}\left[\left(\frac{\Omega_{\mu}}{\omega-\Omega_{\mu}}\right) \nabla_{\|} \ln B\right. \\
& \left.\left.+\nabla_{\|} \ln N_{\mu}\right]\left(n_{\mu}^{2}-1\right)\right\} .
\end{aligned}
$$

The equations (7), (14)-(16), (19), (20) make the closed system of equations of the one dimensional steady state quasihydrodynamics with taking into account the field aligned ponderomotive force. 


\section{Plasma Density}

\subsection{Balance of forces}

In this and the following two sections we shall use the equations (12) and (20) in studies of the ponderomotive influences on the ions in equatorial zone of the magnetosphere. Let us start with a simple case of hydrostatic equilibrium.

According to (7) the balance of ponderomotive, gravitational and pressure-gradient forces is described by the equation

$$
\nabla_{\|} p=\rho g_{\|}+f_{\|} .
$$

We know that the expression (12) for the ponderomotive force $f_{\|}$applies when the plasma contains the identical ions. Let us denote $i=1$. Then $\rho=N m_{1}, p=2 N T$, and the ponderomotive acceleration $A=f_{\|} / \rho$ equals

$$
\begin{aligned}
A= & -\left(\frac{c E^{2}}{2 B}\right)\left(\frac{\Omega}{\Omega-\omega}\right) \\
& \cdot\left[\left(\frac{\omega}{\Omega-\omega}\right) \nabla_{\|} \ln B+\nabla_{\|} \ln \rho\right],
\end{aligned}
$$

where $\Omega=e B / m_{1} c$. The balance of forces (21) takes the form

$$
c_{s}^{2} \nabla_{\|} \ln \rho=g_{\|}+A,
$$

where $c_{s}=\left(2 T / m_{1}\right)^{1 / 2}$.

Reference to Eqs. (5), (11) shows that

$$
\frac{E(s)}{E(0)}=\left\{\left[\frac{B(s)}{B(0)}\right]^{3} \frac{\rho(0)}{\rho(s)}\left[\frac{\Omega(s)-\omega}{\Omega(0)-\omega}\right]\right\}^{1 / 4},
$$

where $s$ is the coordinate along a given field line, and $E(0)$, $B(0), \Omega(0), \rho(0)$ are the values at a reference point $s=0$. Substitution of (24) into (22) gives

$$
\begin{aligned}
A(s)= & -\left(\frac{c E(0)}{2 B(0)}\right)^{2}\left(\frac{\rho(0) \Omega(s)}{\rho(s)[\Omega(s)-\omega]}\right)^{1 / 2} \\
& \cdot\left[\left(\frac{\omega}{\Omega(s)-\omega}\right) \nabla_{\|} \ln B(s)+\nabla_{\|} \ln \rho(s)\right] .
\end{aligned}
$$

Rewriting Eq. (23) in terms of the result (25) we obtain

$$
\frac{1}{\rho} \frac{d \rho}{d s}=\frac{P(s) \rho^{1 / 2}-\epsilon Q(s)}{\rho^{1 / 2}+\epsilon R(s)},
$$

where

$$
\begin{gathered}
Q(s)=\frac{[\omega / \Omega(s)][\rho(0)]^{1 / 2}}{(1-v)^{1 / 2}[1-\omega / \Omega(s)]^{3 / 2}} \frac{d}{d s} \ln B(s), \\
P(s)=\frac{g_{\|}(s)}{c_{s}^{2}}, R(s)=\left[\frac{\rho(0)}{(1-v)[1-\omega / \Omega(s)]}\right]^{1 / 2}, \\
v=\frac{\omega}{\Omega(0)}, \epsilon=\left[\frac{c E(0)}{2 c_{s} B(0)}\right]^{2} .
\end{gathered}
$$

\subsection{Redistribution of plasma density}

To seek the solutions $\rho(s)$ of the Eq. (26), there is a need to specify the model of geomagnetic field $\mathbf{B}$, to select the geomagnetic field line, and to prescribe the values $\rho(0), c_{s}$, $\epsilon, \nu$. (Here the parameter $\epsilon$ should not be confused with the dielectric permeability.) It is not difficult to find the solutions by using the numerical methods, but we prefer the analytical investigation since our prime interest here is with the qualitative dependence of the solutions on the governing parameters $\epsilon, v$ and $c_{s}$. Then we will have a general grasp of the plasma redistribution under the action of ponderomotive forces.

Let us consider the properties of the functions $P(s), Q(s)$ and $R(s)$ (see (26)). We place the reference point $s=0$ at one of the local minimums of the function $B(s)$, i.e., $d B / d s=0$, $d^{2} B / d s^{2}>0$ at $s=0$. Let us assume that this minimum is located at the magnetic equator. (This holds true, for example, in the case of magnetic dipole.) Then $g_{\|}(0)=0$. It will be noted that at the periphery of dayside magnetosphere there are two minimums of the magnetic field intensity, and both are placed outside the equator. In such a situation $g_{\|}(0) \neq 0$; however, at the periphery of the magnetosphere, the gravitational force is small and can be neglected at all.

So, we take $g_{\|}(0)=0$. It is evident that $g_{\|}(s)$ is an odd function, i.e., $g_{\|}(-s)=-g_{\|}(s)$, and $\left(\partial g_{\|} / \partial s\right)>0$ at $s=0$. Hence the $P(s)$ is a monotone increasing function which pass through the zero and has the parity $P(-s)=$ $-P(s)$. The function $Q(s)$ is also odd $(Q(-s)=-Q(s))$, and $Q(0)=0$ since $d B / d s=0$ at the equator. However, $Q(s)$ is a nonmonotonic restricted function in contrast to $P(s) . R(s)$ is a real function since $v<1, \omega<\Omega(s)$. This is a positive defined and decreasing function with a maximum at $s=0$. It will be noted also that $R(s)$ is an even function: $R(-s)=R(s)$. Therefore, the denominator in the righthand side of Eq. (26) is an strictly positive and restricted function of $s$. In view of these results, reference to Eq. (26) shows that $\rho(-s)=\rho(s)$.

Before beginning the general analysis of the solutions, it would be well to consider three limiting cases. At first let $\epsilon=0$ (the waves are absent). Upon integrating (26) we obtain (27)

$$
\rho(s)=\rho(0) \exp \left[\frac{1}{c_{s}^{2}} \int_{0}^{s} g_{\|}(s) d s\right] .
$$

In the small vicinity of the equator we have

$$
\rho(s) \approx \rho(0) \exp \left(\frac{\gamma s^{2}}{2 c_{s}^{2}}\right),
$$

where $\gamma=d g_{\|} / d s$ at $s=0$. We can see that without the waves the plasma density has a minimum at the equator.

Now, let $\epsilon \neq 0$, and $v \rightarrow 0$ (low-frequency limit). Then

$$
\ln \left[\frac{\rho(s)}{\rho(0)}\right]+2 \epsilon\left[1-\sqrt{\frac{\rho(0)}{\rho(s)}}\right]=\frac{1}{c_{s}^{2}} \int_{0}^{s} g_{\|}(s) d s .
$$

The qualitative behavior of $\rho(s)$ in the small vicinity of the equator looks like (28), except that the value

$$
c_{s}^{2}+[c E(0) / 2 B(0)]^{2}
$$


is used as the substitute for sound velocity $c_{s}^{2}$. In other words, in the low-frequency limit the ponderomotive force does not change the monotonic decrease in plasma density as we approach the magnetic equator.

At last, let $\epsilon \neq 0$, and nominally $v \rightarrow 1$ (cyclotron resonance limit). In consequence of this formal transition we obtain the asymptotic estimation

$$
\rho(s) \sim\left[\frac{\Omega(0)-\omega}{\Omega(s)-\omega}\right] \rho(0) .
$$

It is notable that in this case the plasma distribution is independent of the gravity, pressure, and even of the wave amplitude. We can see the steep rise in plasma density as we approach the equator. The width of peak is proportional to $\sqrt{1-v}$; it tends to zero as $v \rightarrow 1$. Really, let us invoke the parabolic approximation of geomagnetic field, so that

$$
\Omega(s)=\Omega(0)\left[1+(s / a)^{2}\right],
$$

where $a=(2 B)^{1 / 2}\left(\partial^{2} B / \partial s^{2}\right)^{-1 / 2}$ at $s=0$. Then $\rho(s) \sim$ $\rho(0)\left[1+(s / b)^{2}\right]^{-1}$, where $b=a \sqrt{1-v}$ is the width of peak. In reality the point $v=1$ can never be attained due to the resonance absorption of the waves. Nonetheless, (1 $v)_{\min } \sim\left(c_{S} / c_{A}\right)^{3 / 2}$ (e.g., Guglielmi and Pokhotelov, 1996), and therefore $b_{\min } \sim a\left(c_{s} / c_{A}\right)^{3 / 4} \ll a$.

We now turn to a general examination of the integral curves of Eq. (26). Reasoning from the properties of $P, Q$ and $R$ we state that zeros and poles of $\rho(s)$ are absent. Hence the possible extremes of $\rho(s)$ lie on the plane $(s, \rho)$ along the curve

$$
\rho_{c}(s)=[\epsilon Q(s) / P(s)]^{2}
$$

and along the line $s=0$. (These are the so-called zeroisoclines.) With the proviso that $\rho>0$ the other nominal possibility (the axis $s$ ) is ruled out. It is easy to check that $\rho_{c}(s)$ is an even, restricted and strictly positive function with the maximum

$$
\begin{gathered}
\rho_{c}(0)=\left(\epsilon / \epsilon_{c}\right)^{2} \rho(0), \\
\epsilon_{c}=\lim _{s \rightarrow 0}(\sqrt{\rho} P / Q) \\
=\left(\frac{\partial g_{\|}}{\partial s}\right)_{0}\left(\frac{1}{B} \frac{\partial^{2} B}{\partial s^{2}}\right)_{0}^{-1} \frac{(1-v)^{2}}{c_{s}^{2} v}
\end{gathered}
$$

at the equator. Here inferior index " 0 " denotes the point $s=0$. The curve (32) and line $s=0$ separate the region of physically admissible values of $\rho$ and $s$ into four domains with a definite sign of $d \rho / d s$ in the each domain in the manner shown in Fig. 1. This information suffices to describe the qualitative behavior of the integral curves.

As long as $\epsilon<\epsilon_{c}, \rho(s)$ has a single extremum, namely, the minimum at $s=0$. The limiting cases $\epsilon=0$ and $\nu \rightarrow 0$ discussed above are in this category. As soon as $\epsilon$ exceeds the critical value $\epsilon_{c}$, three extremes appears on the curve $\rho(s)$, namely, the maximum at $s=0$ and two minimums which lie along the separatrix (32). Thus, the plasma density distribution changes qualitatively as the governing parameter $\epsilon$ passes from $\epsilon<\epsilon_{c}$ to $\epsilon>\epsilon_{c}$. This transition happens at the critical value of the wave amplitude

$$
E_{c}=E_{*}(\Omega-\omega) /(\Omega \omega)^{1 / 2} .
$$

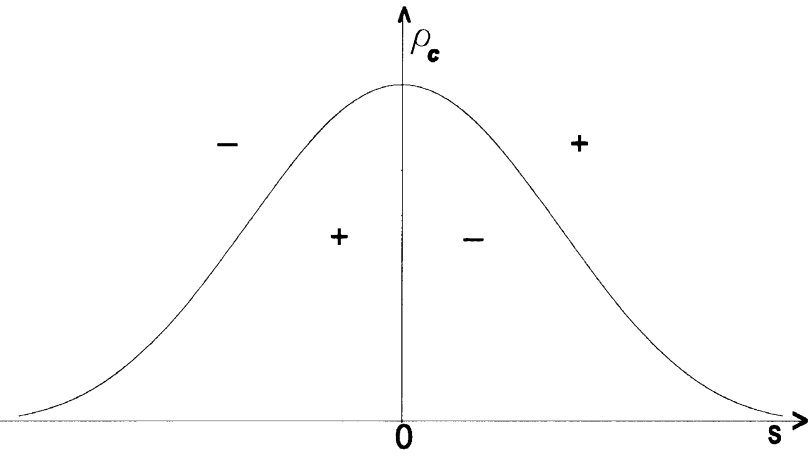

Fig. 1. The separatrix $\rho_{c}(s)$ and the line $s=0$ break the half-plane $s, \rho$ into four domains with a definite sign of $d \rho / d s$ in the each domain. The possible extremes of the integral curves $\rho(s)$ lie along the line $s=0$ and/or along the separatrix.

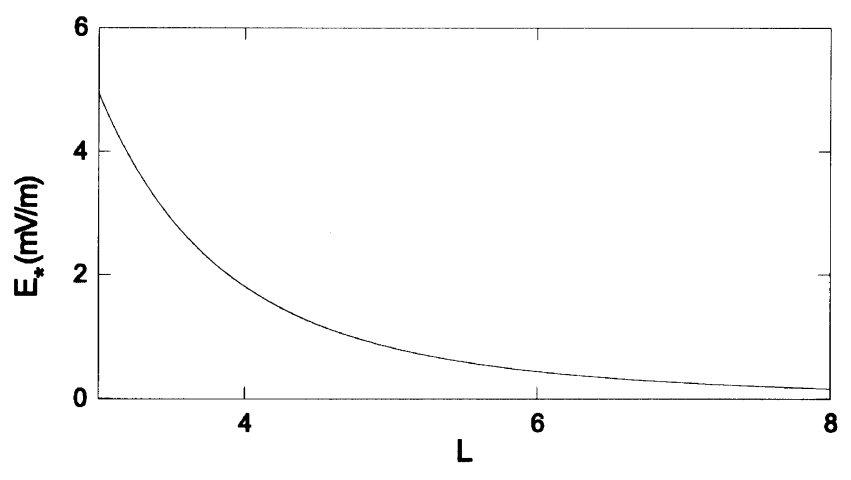

Fig. 2. The $L$-dependence of $E_{*}$ in the dipole magnetosphere (see Eq. (36)).

Here the meaning of $E_{*}$ is evident from comparison of (34) and (35). In the case of dipole approximation of the geomagnetic field we have

$$
E_{*}=\frac{2 \sqrt{2}}{3} \frac{B_{E}\left(R_{E} g_{E}\right)^{1 / 2}}{c L^{3 / 2}},
$$

where $B_{E}=0.31 \mathrm{G}, R_{E}=6.4 \cdot 10^{8} \mathrm{~cm}, g_{E}=980 \mathrm{~cm} / \mathrm{s}^{2}$, and $L$ is the McIlwain parameter. Figure 2 shows the $L$ dependence of $E_{*}$. Figure 3 shows the demarcation line in the plane of governing parameters $\omega / \Omega$ and $E / E_{*}$. If a point is situated to the left (right) of demarcation line, then the plasma density has a minimum (maximum) at the magnetic equator. As an illustration, Figure 4 shows two representative distributions of the plasma density. We can see the qualitative change in the plasma density distribution as the wave amplitude $E$ passes from $E<E_{c}$ (curve $a$ ) to $E>E_{c}$ (curve $b$ ). It should be particularly emphasised that Figures 2-4 are plotted with allowance made for the competing effects (pressure, gravity, ambipolar electric field) and not just the ponderomotive effect.

As an illustration we consider the Pc 1 magnetic pulsation which was detected by Bossen et al. (1976) at the geosynchronous orbit. According to Fig. 1 of their work, the amplitude of magnetic field oscillations is of the order to $b \simeq 3.5$ $\mathrm{nT}$ at the frequency $0.25 \mathrm{~Hz}$. Instead of relations (35), (36), 


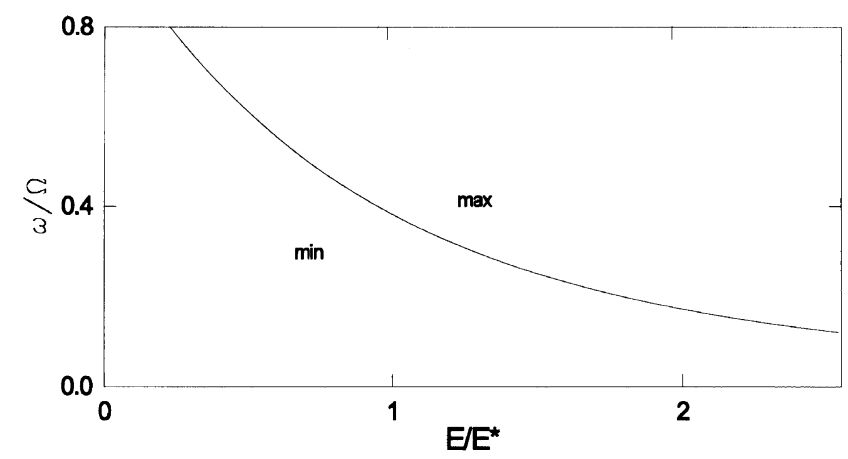

Fig. 3. The demarcation line in the plane of governing parameters $\omega / \Omega$ and $E / E_{*}$. If a point is situated to the left (right) of demarcation line, then the plasma density have a minimum (maximum) at the magnetic equator.

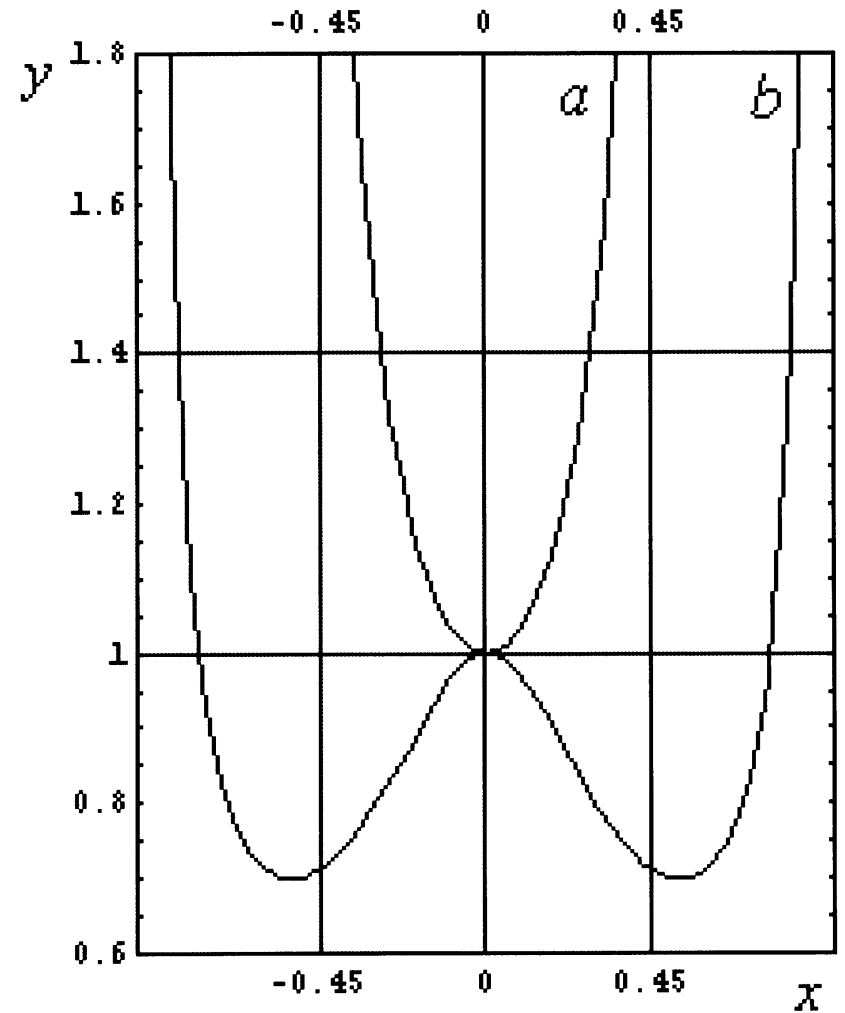

Fig. 4. The distribution of the normalized plasma density $y(x)=\rho(x) / \rho(0)$ along the dipole magnetic field line versus $x=\sin \phi$, where $\phi$ is the latitude. Here $L=5, v=0.5, E_{*}=0.83 \mathrm{mV} / \mathrm{m}, E_{c}=0.58 \mathrm{mV} / \mathrm{m}$ (see the text). Figure illustrates the qualitative change in the plasma density distribution $\rho(x)$ as the wave amplitude $E$ increases from $E<E_{c}$ (curve $a, E=0.2 \mathrm{mV} / \mathrm{m}$ ) to $E>E_{c}$ (curve $b, E=2 \mathrm{mV} / \mathrm{m}$ ).

the following equivalent relations

$$
\begin{gathered}
b_{c}=b_{*}[(\Omega-\omega) / \omega]^{1 / 2}, \\
b_{*}=\left(32 \pi \rho R_{E} g_{E} / 9 L\right)^{1 / 2}
\end{gathered}
$$

can be used in this occasion. At $L=6.6$ we have $\omega / \Omega=$ 0.15 in the dipole magnetic field. If we use the characteristic value $\rho=3 \cdot 10^{-20} \mathrm{~kg} / \mathrm{m}^{3}$, then $b_{c} \simeq 1.34 \mathrm{nT}$. Hence $b / b_{c} \simeq$ 2.6 , i.e., we expect the distribution of plasma density with a maximum at the equator.

Let us return to the limiting case $v \rightarrow 1$ discussed above (see Eq. (31)). It is evident that we are dealing here with the

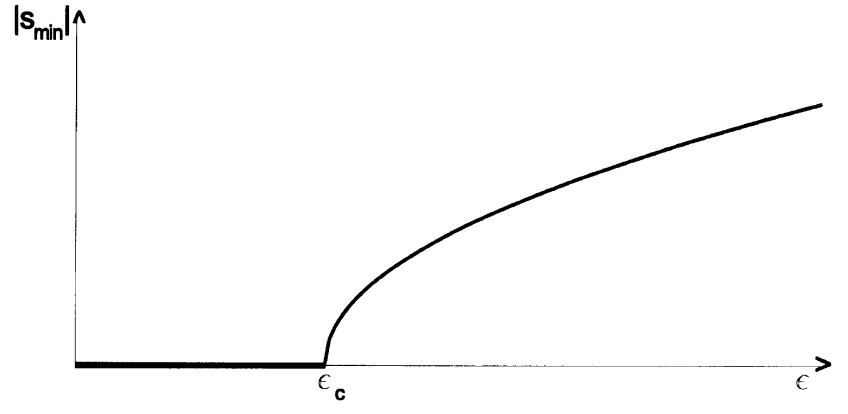

$[\mathrm{t}]$

Fig. 5. The bifurcation diagram. The thick line gives the position $s_{\min }$ of $\rho_{\min }$ as a function of the governing parameter $\epsilon$. Above the threshold $\epsilon_{c}$ the value $\left|s_{\min }\right|$ is proportional to the square root of supercriticality.

case of $E>E_{c}$. If $v \rightarrow 1$, then $E_{c} \rightarrow 0$ in accordance with our conclusion that the distribution (31) does not depend on the wave amplitude. However, in reality $(1-v)_{\min } \sim$ $\left(c_{S} / c_{A}\right)^{3 / 2}$, so that $E_{c \text { min }} \sim E_{*}\left(c_{S} / c_{A}\right)^{3 / 2}$ as long as $c_{S} \ll c_{A}$.

The ponderomotive redistribution of the plasma density in going from state $\epsilon<\epsilon_{c}$ to state $\epsilon>\epsilon_{c}$ closely resembles phase transition of the second kind. Let us denote the position of $\rho_{\min }$ by $s_{\min }$. We take $\left|s_{\min }\right|$ as an order parameter. At $\epsilon<\epsilon_{c}$ we have $s_{\min }=0$. When crossing the threshold $\left(\epsilon>\epsilon_{c}\right)$ the system exhibits a transition of the second kind, and in the line with this assumption

$$
s_{\min } \propto \pm \sqrt{\epsilon-\epsilon_{c}},
$$

i.e., the order parameter is proportional to the square root of supercriticality (e.g., Gilmore, 1981). Figure 5 illustrates the situation. The analysis of Eq. (26) with taking into account the properties of functions $P(s), Q(s)$ and $R(s)$ leads to the estimation

$$
s_{\min }^{2}=4\left(\frac{1-v}{2+v}\right)\left(\frac{1}{B} \frac{\partial^{2} B}{\partial s^{2}}\right)_{0}^{-1}\left(\frac{\epsilon}{\epsilon_{c}}-1\right)
$$

near the threshold. This result substantiates our hypothesis (37).

\section{Capture of Heavy Ions \\ 4.1 Equation of motion}

Going to the case of multicomponent plasma, we face two problems. Both of these problems are directly related to the changes in the spatial structure of the refractive index. One difficulty concerns the appearance of zeros and so-called crossovers of $n^{2}$ (e.g., Guglielmi and Pokhotelov, 1996). The WKB approximation is not valid in the vicinity of corresponding points along the wave path. Hence, we can not use the equation (5) to describe a spatial structure of the wave field in relation to the distribution of ions, which in turn is conditioned by the wave structure. It seems likely that in the general case there is no way to study the ponderomotive redistribution of ions in the multicomponent plasma without recourse to numerical integration of the nonlinear wave equation. However, this is a challenging task. In this and the next sections we shall consider some special cases when it is tolerable to use the Equation (5) for at least qualitative description of the wave field. 
The second problem is indirectly related to the additional poles of the refractive index in the multicomponent plasma. The poles of $n^{2}$ at the gyrofrequencies of heavy ions (see Eq. (9)) correlate with the corresponding singularities appearing in the expression of the ponderomotive force (see Eq. (18)). This involves a considerable challenge of a fundamental nature. The generalization of the equations presented in Subsection 2.2 is demanded to eliminate these singularities. Moreover, strictly speaking the concept of averaged ponderomotive force, as such, is liable to break down in the vicinity of gyroresonances of the heavy ions. Here many questions still remain to be answered. Nevertheless, an attempt must be made to use our present notion of ponderomotive force after an elimination of infinities.

We begin with the one-dimensional field-aligned motion of the heavy "test particle", i.e., a single ion $i=2$ in the plasma containing the one kind of ions $i=1$ with $m_{2}>m_{1}$. In this case, there is no the first difficulty mentioned above. Equation of motion of the test particle is

$$
\dot{v}=g_{\|}+\frac{e}{m_{2}} E_{\|}+A_{2},
$$

where the ambipolar electric field is equal to

$$
E_{\|}=-\left(\frac{T}{e}\right) \nabla_{\|} \ln \rho
$$

and the ponderomotive acceleration equals

$$
\begin{aligned}
A_{2}= & \frac{e^{2} E^{2}}{2 m_{2}^{2} \omega\left[\left(\Omega_{2}-\omega\right)^{2}+\delta^{2}\right]} \\
& \cdot\left[\left(\Omega_{2}-\omega\right) \nabla_{\|} \ln E^{2}-\Omega_{2} \nabla_{\|} \ln B\right] .
\end{aligned}
$$

Here $\delta$ is an effective collision frequency (see Appendix), and $\rho=m_{1} N$. Substitution of (5) into (40) yields

$$
\begin{aligned}
A_{2}= & -\frac{e^{2} E^{2}\left(\Omega_{2}-\omega\right)}{2 m_{2}^{2} \omega\left[\left(\Omega_{2}-\omega\right)^{2}+\delta^{2}\right]} \\
& \cdot\left[\left(\frac{\omega}{\Omega_{2}-\omega}\right) \nabla_{\|} \ln B+\frac{1}{2} \nabla_{\|} \ln n^{2}\right] .
\end{aligned}
$$

This coincides with (18) if $\delta=0$. Substitution of (11) with $i=1$ in (41) yields

$$
\begin{aligned}
A_{2}= & -\frac{e^{2} E^{2}\left(\Omega_{2}-\omega\right)}{4 m_{2}^{2} \omega\left[\left(\Omega_{2}-\omega\right)^{2}+\delta^{2}\right]} \\
& \cdot\left[\left(\frac{2 \omega}{\Omega_{2}-\omega}-\frac{2 \Omega_{1}-\omega}{\Omega_{1}-\omega}\right) \nabla_{\|} \ln B+\nabla_{\|} \ln \rho\right],
\end{aligned}
$$

and lastly from (38), (39), (42) it follows that

$$
\begin{aligned}
\frac{d v}{d t}= & g_{\|}-\left[\frac{T}{m_{2}}+\frac{e^{2} E^{2}\left(\Omega_{2}-\omega\right)}{4 m_{2}^{2} \omega\left[\left(\Omega_{2}-\omega\right)^{2}+\delta^{2}\right]}\right] \nabla_{\|} \ln \rho \\
& -\frac{e^{2} E^{2}\left(\Omega_{2}-\omega\right)}{4 m_{2}^{2} \omega\left[\left(\Omega_{2}-\omega\right)^{2}+\delta^{2}\right]} \\
& \cdot\left(\frac{2 \omega}{\Omega_{2}-\omega}-\frac{2 \Omega_{1}-\omega}{\Omega_{1}-\omega}\right) \nabla_{\|} \ln B .
\end{aligned}
$$

In relation to this equation some remarks are in order. We used the equation (11) for the square of refractive index in the collisionless plasma $(\delta=0)$. This approximation for $n^{2}$ is justified in the following regime: $\delta \ll \omega, \delta^{2} \ll\left(\Omega_{1}-\omega\right)^{2}$. Hence the term $\delta^{2}$ in Eq. (43) has a role exclusively at the frequencies $\omega \sim \Omega_{2}$. In fact, we have introduced the collisions to nothing more than eliminate the infinity appearing in the expressions of ponderomotive effects at the gyrofrequency $\Omega_{2}$. For the same reason we do not include the effect of collisions in the equation of motion (38). In the end of this section we shall have more to say about Eq. (43).

One additional comment is necessary. In general, when writing the equations of motion, account must be taken of the mirror force term (e.g., Northrop, 1963). We drop this term on the right sides of Eqs. (38) and (43) for the sake of simplicity. In other words, we restrict our consideration to the specific case of the test particles with zero magnetic moment in the absence of the wave. We would like to note that the particle in the wave field gains an effective magnetic moment resulting in appearance of the mirror force. However, this wave induced mirror force is already properly accounted in the Eqs. (40) - (43). Of course, the effects of "usual" mirror force may also be allowed for as the need arose, but here we prefer to study the ponderomotive forces in the framework of simple model. Note also that the similar approach is used by Hultqvist (1996): he assumes that the particle magnetic moment equals zero in the absence of the waves.

\subsection{Equator as an attractor for heavy ions}

Let us introduce the ponderomotive potential $U(s)$ to bring the equation (43) into the form:

$$
\frac{d v}{d t}=-\frac{\partial U}{\partial s} .
$$

Here $s$ is the coordinate along the geomagnetic field lines as previously. The conservation of energy gives

$$
v= \pm \sqrt{2[W-U(s)]},
$$

where $W$ is a constant. We can see that our test particle moves within the domains where $U(s)<W$. (The value $W$ is the total energy of test particle per unit mass.)

The analysis of the right-hand side of (43) enables us to present roughly the general view of $U(s)$ at the frequencies $\omega \sim \Omega_{2}$ in the vicinity of magnetic equator (Fig. 6). We can see that the equator is the attractor for heavy ion. If $W>U_{m}$, the particle executes an infinite motion; if $W<$ $U_{m}$, the particle executes a finite motion, i.e., it is trapped in the potential trough of depth $U_{m}$. The region of motion of captured ion is confined to the interval $\left(-s_{0}, s_{0}\right)$. The borderpoints $\pm s_{0}$ are defined by the equation

$$
U\left(s_{0}\right)=W .
$$

The period of the finite motion

$$
T=2 \sqrt{2} \int_{0}^{s_{0}} \frac{d s}{\sqrt{W-U(s)}}
$$

depends on the wave frequency $\omega$, wave amplitude $E$, together with the parameter of motion $W$. 


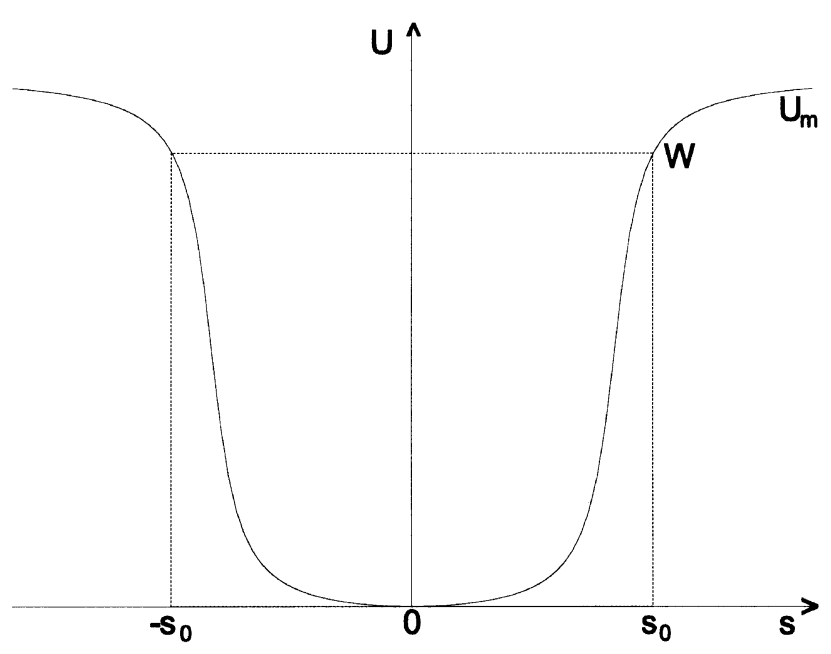

Fig. 6. The general view of the ponderomotive potential $U(s)$ at the resonant frequencies $\left(\omega \sim \Omega_{2}\right)$ in the vicinity of magnetic equator. The region of motion of a heavy ion is confined to the interval $\left(-s_{0}, s_{0}\right)$. Here $W$ is the constant of motion.

To make an estimate of $T$, let us consider the potential

$$
\begin{aligned}
U(s)=\frac{e c E^{2}}{2 m_{2} B \delta} & {\left[\arctan \left(\frac{\omega-\Omega_{2}(0)}{\delta}\right)\right.} \\
& \left.-\arctan \left(\frac{\omega-\Omega_{2}(s)}{\delta}\right)\right] .
\end{aligned}
$$

It corresponds to the main term in the right-hand side of (43) at the resonant frequencies $\omega \approx \Omega_{2}$. The depth of the potential well equals

$$
U_{m}=\frac{e c E^{2}}{2 m_{2} B \delta}\left[\frac{\pi}{2}+\arctan \left(\frac{\omega-\Omega_{2}(0)}{\delta}\right)\right] .
$$

It is easy to check that

$$
U_{m}<\frac{\pi \omega}{2 \delta}\left(\frac{c E}{B}\right)^{2}
$$

Let us assume that $\Omega_{2}(0)<\omega$, and $\delta \ll \omega-\Omega_{2}(0)$. Then with the reference to Eqs. (47), (48) it can be seen that

$$
T \approx \frac{4 a}{v}\left(1-\frac{\Omega_{2}(0)}{\omega}\right)^{1 / 2} .
$$

Here $v=\sqrt{2 W}$, and

$$
a=\left(\frac{1}{2 B} \frac{\partial^{2} B}{\partial s^{2}}\right)_{0}^{1 / 2}
$$

or $a=(\sqrt{2} / 3) R_{E} L$ in the case of dipole field. From (51) we notice that the period $T$ depends on the energy of motion $W$; that is to say that the captured ion executes the nonharmonic motion. The harmonic oscillations with the period

$$
T \approx(2 \pi a / c)(B \delta / E \omega)
$$

take place when $\omega \rightarrow \Omega_{2}(0)$, and $W \ll U_{m}$. Here $B=$ $B_{E} / L^{3}$.
It will be recalled that $v<\sqrt{2 U_{m}}$. Once more restriction on the velocity follows from the fact that the Doppler effect is disregarded in Eq. (43). Ignoring this effect is permissible for the nonresonant particles with the proviso that $v$ is much less than the phase velocity of the wave. In the case of resonant particles this condition is inadequate. However, rigorous consideration of the Doppler shift demands a revision of the theory of ponderomotive forces outlined in Section 2. This is beyond the scope of our present work.

At last, the challenging question relates to the effective collision frequency $\delta$. We have introduced $\delta$ exclusively for the regularization of the ponderomotive acceleration (40) at the frequencies $\omega \sim \Omega_{2}$. In the ionosphere the collision term has the literal sense, so that the equation (40), as such, finds use. However, in the magnetosphere the physical meaning of $\delta$ should yet be search for. Guglielmi and Lundin (1999) have presented a heuristic rule which can be termed as "sizeregularization". This name come from the fact that we insert a characteristic transversal size $l_{\perp}$ of the wave field into the model. The relation $\delta \sim\left(c / l_{\perp}\right)(E / B)$ is set by recognizing that the displacement of the ion under the action of the wave field is no more than $l_{\perp}$. If so, then (52) gives $T \sim 2 \pi a / \omega l_{\perp}$.

\section{Binary Mixture of Ions}

\subsection{Equations of equilibrium}

Let us consider the diffusion equilibrium of ions in a multicomponent plasma. Putting $u_{i}=0$, we obtain from (14), (16), (17):

$$
\begin{gathered}
c_{i}^{2} \nabla_{\|} \ln \left(N_{i} \sum_{j} N_{j}\right)=g_{\|}+A_{i}, \\
A_{i}=\frac{e^{2} E^{2}}{2 m_{e} m_{i}\left(\Omega_{e}+\omega\right)\left(\Omega_{i}-\omega\right)} \\
\cdot\left[\nabla_{\|} \ln E^{2}-\frac{\Omega_{e}\left(2 \Omega_{i}-\omega\right)}{\left(\Omega_{e}+\omega\right)\left(\Omega_{i}-\omega\right)} \nabla_{\|} \ln B\right] .
\end{gathered}
$$

Here $c_{i}=\left(T / m_{i}\right)^{1 / 2},\{i, j\}=1,2, \ldots, m_{e}<<m_{i}$. It is preferable to use

$$
\begin{aligned}
A_{i}= & -\left(\frac{c E}{2 B}\right)^{2}\left(\frac{2 \Omega_{i}}{\Omega_{i}-\omega}\right) \\
& \cdot\left[\left(\frac{\Omega_{i}}{\Omega_{i}-\omega}\right) \nabla_{\|} \ln B+\frac{1}{2} \nabla_{\|} \ln \left(\sum_{j} \frac{\omega_{0 j}^{2}}{\Omega_{j}\left(\Omega_{j}-\omega\right)}\right)\right]
\end{aligned}
$$

rather than (54). It follows from (20) at the frequencies much less then the electron gyrofrequency, $\omega \ll \Omega_{e}$.

The equations (53), (55) together with (5), (9), (16) represent the closed system of quasi-linear equations. This is reasonably cumbersome system. For simplicity we restrict analysis to the case of binary mixture of ions, i.e., $\{i, j\}=1,2$. Then the square of refractive index (9) equals

$$
n^{2}=1+\frac{\omega_{01}^{2}}{\Omega_{1}\left(\Omega_{1}-\omega\right)}+\frac{\omega_{02}^{2}}{\Omega_{2}\left(\Omega_{2}-\omega\right)},
$$

and the quasi-neutrality condition (16) is $N_{1}+N_{2}=N$.

For definiteness, we shall put $m_{1}<m_{2}$. In other words, we shall consider the two-component plasma involving a mixture 
of the light $(i=1)$ and heavy $(i=2)$ ions. Then $n^{2}=0$ at the frequency $\omega=\Omega_{2}+\Delta \omega$, where approximately

$$
\Delta \omega \approx \frac{N_{2}}{N}\left(\Omega_{1}-\Omega_{2}\right)
$$

in the event that $\Omega_{1}^{2} \ll \omega_{01}^{2}$. As we have mentioned in Section 4 , our theory is not valid in the vicinity of corresponding points along the wave path.

There are two line to get round the difficulty. First, we can restrict our consideration to the frequencies $\omega<\Omega_{2 \min }$; in this case the zeros of $n^{2}$ are absent along the wave path at all. Second, we are entitled to neglect zeros of $n^{2}$ associated with heavy ions on condition that the band $\Delta \omega$ is small enough (e.g., Guglielmi and Pokhotelov, 1996). The theory is not sufficiently advanced to indicate the corresponding validity criterion, but in any case the necessary condition is $N_{2} \ll N$ (see (57)).

\subsection{Equatorial condensation of heavy ions}

From this point on we shall use the ratio $N_{2} / N$ as a small parameter when solving the equations of equilibrium. In other words, it is suggested that the heavy ions constitute a small admixture to the light ions. This corresponds roughly to the magnetospheric conditions at least in a relatively quiescent state.

With the small parameter in hand it becomes possible to solve the quasi-linear equations (53) via step-by-step method. Zeroth-order approximation gives the distribution of light ions. It is akin to the previously described distribution of the single-component plasma (see Section 3). In the first approximation we get the equation descriptive of the distribution of heavy ions:

$$
\begin{aligned}
& \nabla_{\|} \ln N_{2}=\frac{m_{2}}{T} g_{\|} \\
& -\left[1+\frac{m_{2}}{T}\left(\frac{c E}{2 B}\right)^{2}\left(\frac{\Omega_{2}}{\Omega_{2}-\omega}\right)\right] \nabla_{\|} \ln N_{1} \\
& -\frac{m_{2}}{T}\left(\frac{c E}{2 B}\right)^{2} \frac{\omega \Omega_{2}}{\left(\Omega_{2}-\omega\right)^{2}}\left[\frac{\Omega_{1}-\Omega_{2}}{\Omega_{1}-\omega}+1\right] \nabla_{\|} \ln B .
\end{aligned}
$$

Upon integrating (58) we obtain

$$
N_{2}(s)=N_{2}(0) \exp \left[\frac{m_{2}}{T} \int_{0}^{s} \alpha(s) d s\right],
$$

where

$$
\begin{aligned}
\alpha= & \frac{\left(2 c_{1}^{2}-c_{2}^{2}\right)\left(\Omega_{1}-\omega\right)\left(\Omega_{2}-\omega\right)-2 c_{1}^{2} \epsilon \omega\left(\Omega_{1}-\Omega_{2}\right)}{2 c_{1}^{2}\left(\Omega_{2}-\omega\right)\left(\Omega_{1}-\omega+\epsilon \Omega_{1}\right)} g_{\|} \\
& -\frac{2 c_{1}^{2} \epsilon \omega}{\left(\Omega_{1}-\omega\right)\left(\Omega_{2}-\omega\right)} \\
& \cdot\left[1+2\left(\frac{\Omega_{1}-\Omega_{2}}{\Omega_{2}-\omega}\right)-\frac{1}{2}\left(\frac{\Omega_{2}-\omega+2 \epsilon \Omega_{1}}{\Omega_{1}-\omega+\epsilon \Omega_{1}}\right)\right] \frac{\partial \Omega_{2}}{\partial s} .
\end{aligned}
$$

Here $s$ denotes the coordinate along geomagnetic field line with $s=0$ at the equator, $\epsilon=\left(c E / 2 c_{s} B\right)^{2}$ is the normalized wave intensity, $c_{s}=\left(2 T / m_{1}\right)^{1 / 2}$ is the sound velocity.

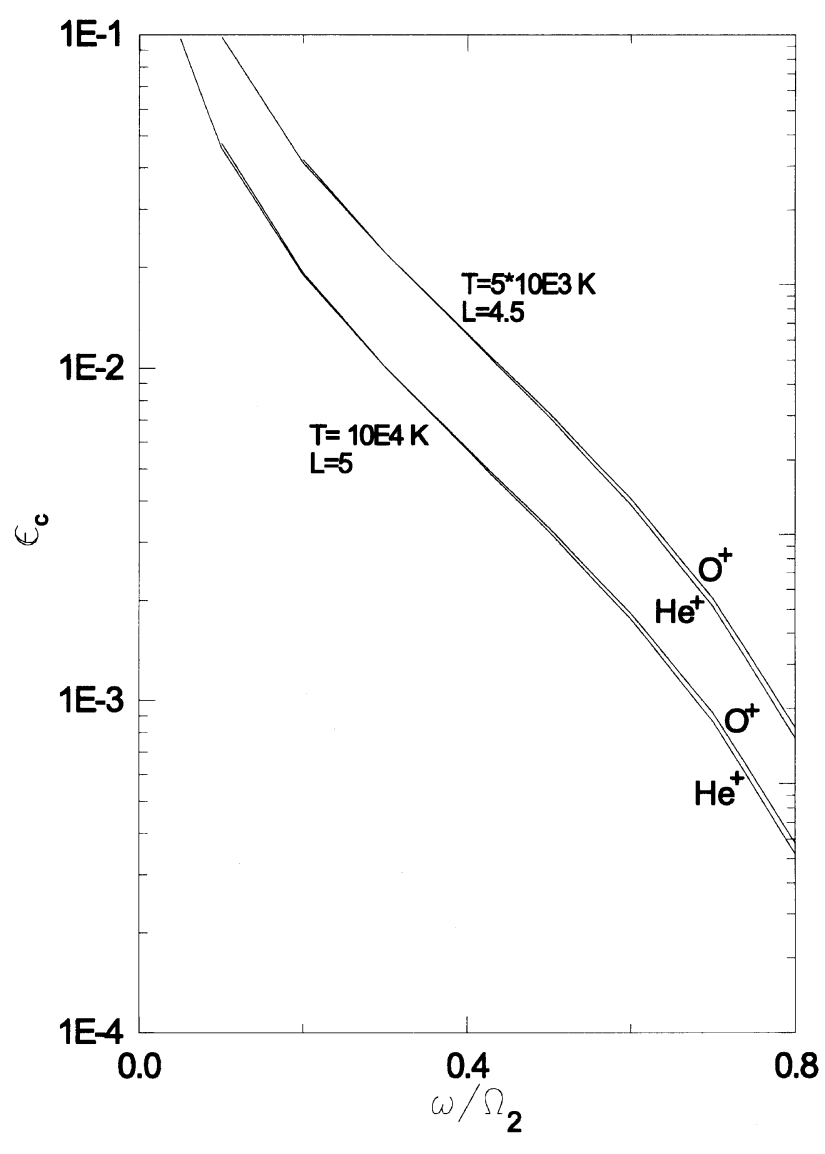

Fig. 7. The critical value of normalized wave intensity as a function of the normalized wave frequency. Providing that $\epsilon>\epsilon_{c}$, the heavy ions collect near the magnetic equator. Here $T=5 \cdot 10^{3} \mathrm{~K}\left(10^{4} \mathrm{~K}\right)$ and $L=4.5(5)$ for the upper (lower) couple of curves. The upper (lower) curve in each couple corresponds to plasma with $\mathrm{O}^{+}\left(\mathrm{He}^{+}\right)$ion admixture (see the text).

The following assay is similar to that described in Subsection 3.2. The main result is illustrated in Fig. 7. Here we can see the critical value of normalized wave intensity $\epsilon_{c}$ as a function of the normalized wave frequency $\omega / \Omega_{2}$. The curves labeled $\mathrm{He}^{+}\left(\mathrm{O}^{+}\right)$correspond to the case $m_{2}=4 m_{1}$ $\left(m_{2}=16 m_{1}\right)$, i.e., the case of small admixture of helium (oxygen) ions in the hydrogen plasma. Here $T=5 \cdot 10^{3} \mathrm{~K}$ $\left(10^{4} \mathrm{~K}\right)$ and $L=4.5(5)$ for the upper (lower) couple of curves. The upper (lower) curve in each couple corresponds to plasma with $\mathrm{O}^{+}\left(\mathrm{He}^{+}\right)$ion admixture. As long as $\epsilon<\epsilon_{c}$, the concentration of heavy ions $N_{2}(s)$ has the minimum at $s=0$, otherwise $N_{2}(s)$ has the maximum at $s=0$. In other words, the heavy ions collect near the magnetic equator with providing that $\epsilon>\epsilon_{c}$.

\section{Summary and Discussion}

The principal contributions of this paper are as follows.

(1) The presentation of the equations describing the ponderomotive influence of ion cyclotron waves on the magnetospheric ions, and, more importantly,

(2) the demonstration of manifestations of the ponderomotive redistribution of ions in the equatorial zone of the magnetosphere.

Let us now see what we can learn in general sense from our investigation. In the first place, we can definitely con- 
clude that the field-aligned ponderomotive force have a pronounced impact on the ion population provided the waves are comparatively strong. This conclusion conforms with the results obtained previously (e.g., Lundin, 1988; Lundin and Hultqvist, 1989; Allan, 1992; Guglielmi et al., 1996; Hultqvist, 1996). It may be more surprising that the uses of the theory of ponderomotive force in the magnetospheric physics run into obstacles. We can see that the difficulties start with the very formulation of problems. The general phenomenological equations (1), (2) derived by Pitaevsky (1960), Washimi and Karpman (1976) are not suitable for the multicomponent plasma. The use of the quasi-hydrodynamic equations instead of (1), (2) partially has made in improving the situation; however, some problems of the fundamental nature still stand. One of them is the problem of ponderomotive self-action of the waves (Guglielmi, 1992, 1997). The approximate solution (5) of the self-consistency problem is limited in use to the short travelling waves. It fails if zeros of $n^{2}$ associated with heavy ions occur along the wave path in the multicomponent plasma (see Section 5).

The poles of $n^{2}$ at the gyrofrequencies of heavy ions pose the problem too. According to Eqs. (18)-(20), the ponderomotive acceleration tends to infinity if $\omega \rightarrow \Omega_{i}$. This is so-called ponderomotive resonance. In Section 4 we have introduced the effective collision frequency $\delta$ to eliminate the infinity, but this is nothing more than a palliative. Guglielmi and Lundin (1999) have presented an alternative cure for this difficulty; however, in our opinion we have still a long way to go toward the understanding how the heavy ion travels through the region of ponderomotive resonance. This problem is of prime importance for the magnetospheric physics, especially in connection with the satellite observation of upward moving heavy ions (Lundin, 1988; Gustafsson et al., 1990; Lundin et al., 1990; Lundin and Eliasson, 1991). From the most elementary viewpoint, the region where $\omega \sim \Omega_{i}$ is a peculiar kind of effusor in which the high ponderomotive potential converts into the field-aligned velocity.

The results presented in the Section 3 seem to be rest on the more solid ground. It should be remembered that these results were obtained in the framework of time-independent model. Even so, there is little question that the magnetospheric plasma tends to collect in the vicinity of geomagnetic equator under the action of ion cyclotron waves. This tendency will, in the certain conditions, lead to formation of the plasma density distribution with a maximum at the equator. One such condition is the inequality $E>E_{c}$ (see Figs. 2 and 3 ). Other condition is as follows: the ponderomotive force acts during a sufficiently long time in order to establish the stationary state. The corresponding criterion, as it usually is, should be searched for in the context of an advanced theory. Here we can only present a conservative estimate. Let us adopt the value $\tau \sim s_{\min } / c_{s}$ as an order estimation of the time scale for the particle redistribution with an eye to the fact that the real time scale may be greater than $\tau$. Then a necessary condition for feasibility of the steady state approximation is $\delta t>s_{\min } / c_{s}$, where $\delta t$ is the duration of quasi-stationary regime of the electromagnetic oscillations. By way of example, assume that $s_{\min } \sim 2 \cdot 10^{9} \mathrm{~cm}$ and $c_{s} \sim 2 \cdot 10^{6} \mathrm{~cm}$ $\mathrm{s}^{-1}$. Then the necessary condition is $\delta \dot{t}>10^{3} \mathrm{~s}$. The attempt to find the sufficient condition by a simple way has not met success. The ion cyclotron waves are observed in the form of so-called Pcl geomagnetic pulsations. There is an interconnection between the appearance of a large body of Pc1 events and the refilling of plasmaspheric flux tubes at the recovery phase of magnetic storm (e.g., Kangas et al., 1998). It would be of interest to study the possible impact of the ponderomotive force upon the process of plasmasphere refilling. To do this would require a consideration of the nonstationary problems, since as judged from the physical reasoning, the excitation of ion cyclotron $\mathrm{Pcl}$ waves in the equatorial zone of the magnetosphere leads to formation of the rarefaction acoustic shocks which travel along the field lines toward the Earth.

A wide range of questions is connected with the ponderomotive wave-particle interaction at the periphery of the magnetosphere. The main peculiarity of the dayside periphery is that there are two minima of the magnetic field intensity which are located outside the equatorial plane. The bifurcation, i.e., the transition from one minimum to two minima take place at the distance $r \sim 0.8 r_{m}$ away from the Earth, where $r_{m}$ is the distance to the magnetopause. The most ion cyclotron wave events, on the other hand, occurred just on the dayside periphery of the magnetosphere (Anderson, 1992). If so, the theory seemingly predicts the distribution of plasma density with minimum at the equator and two maximums outside the equator at the distances $0.8 r_{m}<r<r_{m}$. However, in fact the theory outlined in the paper ignored the magnetospheric convection which is liable to alter grossly the plasma distribution at the periphery of the magnetosphere.

In the nightside outer magnetosphere, the neutral sheet of geomagnetic tail has aroused interest in the context of presented paper. It follows from general considerations that the ion-cyclotron waves push the plasma towards the neutral sheet. However, as in the case of dayside outer magnetosphere, the theory is unsuitable here in its present form.

In this paper we used the hydrodynamics, quasi-hydrodynamics, and test-particle approaches. The general approach based on the application of kinetic equations is difficult mathematically, but it allows to describe in more detail the ponderomotive redistribution of ions in the magnetosphere. In particular, it allows to study the instabilities which may arise due to this redistribution. The application of method of kinetic equations to the problem of ponderomotive redistribution of ions will be subject of the future publications.

Acknowledgments. We would like to thank B. Aparicio, J. Kangas, K. Stasiewicz, and M. Yamauchi for the interesting discussions.

\section{Appendix. Evaluation of the Equation (40)}

It is shown here how the equation (40) is derived from the equation of motion of a test particle. This is akin to the derivation described by Guglielmi and Lundin (1999), except that the collisions of particle are built into the equation of motion. First, the formula for the magnetic moment

$$
\mu=(e / 2 c)\langle\mathbf{x} \times \mathbf{v}\rangle
$$

is used. Here the angular parenthesis denotes the averaging over the period of electric field oscillations, $\mathbf{x}(t)$ is the trajectory of a particle, $\mathbf{v}=\dot{\mathbf{x}}$. From the equation of motion it 
follows that

$$
x(t)=a \exp (-i \omega t), y(t)=-i a \exp (-i \omega t),
$$

where

$$
a=\frac{e E}{m \omega(\Omega-\omega-i \delta)} .
$$

Here $\delta$ is the collision frequency. Hence the field-aligned component of the magnetic moment is

$$
\mu=-\frac{e^{3} E^{2}}{2 \omega c m^{2}\left[(\Omega-\omega)^{2}+\delta^{2}\right]}
$$

Therefore, the so-called Lundin-Hultqvist force ("magnetic moment pumping") $F_{L H}=\mu \cdot \nabla_{\|} B$ equals

$$
F_{L H}=-\frac{e^{3} E^{2}}{2 m^{2} c \omega\left[(\Omega-\omega)^{2}+\delta^{2}\right]} \nabla_{\|} B .
$$

In a similar manner, the Miller force is derived:

$$
F_{M}=\frac{e^{2}(\Omega-\omega)}{2 m \omega\left[(\Omega-\omega)^{2}+\delta^{2}\right]} \nabla_{\|} E^{2}
$$

The ponderomotive acceleration $A=m^{-1}\left(F_{L H}+F_{M}\right)$ thus equals

$$
\begin{aligned}
A= & \frac{e^{2} E^{2}}{2 m^{2} \omega\left[(\Omega-\omega)^{2}+\delta^{2}\right]} \\
& \cdot\left[(\Omega-\omega) \nabla_{\|} \ln E^{2}-\Omega \nabla_{\|} \ln B\right] .
\end{aligned}
$$

This coincides with (17) if $\delta=0$.

\section{References}

Allan, W., Ponderomotive mass transport in the magnetosphere, J. Geophys. Res., 97, 8483-8493, 1992.

Allan, W., The ponderomotive force of standing Alfvén waves in a dipolar magnetosphere, J. Geophys. Res., 98, 1409-1417, 1993a.

Allan, W., Plasma energization by the ponderomotive force of magnetospheric standing Alfvén waves, J. Geophys. Res., 98, 11383-11390, 1993b.

Allan, W., J. R. Manuel, and E. M. Poulter, Magnetospheric cavity modes: Some nonlinear effects, J. Geophys. Res., 96, 11461-11473, 1991.

Anderson, B. J., Recent observations of electromagnetic ion cyclotron waves in space, Adv. Space. Res., 17, 1041-1044, 1996.

Anderson, B. J., R. E. Erlandson, and L. J. Zanetti, A statistical study of Pc 1-2 magnetic pulsations in the equatorial magnetosphere: 1, Equatorial occurrence distributions, J. Geophys. Res., 97, 3075-3088, 1992a.

Anderson, B. J., R. E. Erlandson, and L. J. Zanetti, A statistical study of Pc 1-2 magnetic pulsations in the equatorial magnetosphere: 2, Wave properties, J. Geophys. Res., 97, 3089-3101, 1992b.

Barghouthi, I. A., Effect of wave-paricle interaction on $\mathrm{H}^{+}$and $\mathrm{O}^{+}$outflow at high latitude: A comparative study, J. Geophys. Res., 102, 2206522015, 1997

Bossen, M., R. L. McPherron, and C. T. Russell, Simultaneous Pc 1 observations by the synchronous satellite ATS-1 and ground stations: implications concerning IPDP generation mechanisms, J. Atmosp. Terr. Phys. 38, 1157-1167, 1976.

Erlandson, R. E. and B. J. Anderson, Pc 1 waves in the ionosphere: A statistical study, J. Geophys. Res., 101, 7843-7857, 1996.

Erlandson, R. E., L. J. Zanetti, T. A. Potemra, L. P. Block, and G. Holmgren, Viking magnetic and electric field observations of Pc 1 waves at high latitudes, J. Geophys. Res., 95, 5941-5955, 1990.

Erlandson, R. E., B. J. Anderson, and L. J. Zanetti, Viking magnetic and electric field observations of periodic Pc 1 waves: Pearl pulsations, $J$. Geophys. Res., 97, 14823-14832, 1992

Fraser, B. J., J. C. Samson, Y. D. Hu, R. L. McPherron, and C. T. Russell, Electromagnetic ion cyclotron waves observed near the oxygen cyclotron frequency by ISEE 1 and 2, J. Geophys. Res., 97, 3063-3074, 1992.
Gilmore, R., Catastrophe Theory for Scientist and Engineers, 666 pp., John Wiley \& Sons, New York, 1981.

Ginzburg, V. L., Propagation of Electromagnetic Waves in a Plasma, 615 pp., Pergamon, New York, 1971.

Ginzburg, V. L., Theoretical Physics and Astrophysics, 416 pp., Nauka, Moskow, 1975 (in Russian).

Guglielmi, A., Ponderomotive forces in the crust and magnetosphere of the Earth, Physics of the Earth, 7, 35-39, 1992 (in Russian).

Guglielmi, A., Comment on the ponderomotive self-action of Alfvén waves, J. Geophys. Res., 102, 209-210, 1997.

Guglielmi, A. V. and O. A. Pokhotelov, Geoelectromagnetic waves, 402 pp. IOP Publishing Ltd, Bristol, 1996.

Guglielmi, A. and R. Lundin, Ponderomotive upward acceleration of ions by ion cyclotron and Alfvén waves over the polar regions, J. Geophys. Res., 105, 2000 (to be published).

Guglielmi, A., O. A. Pokhotelov, F. Z. Feygin, Yu. P. Kurchashov, J. F. McKenzie, P. K. Shukla, L. Stenflo, and A. S. Potapov, Ponderomotive forces in longitudinal MHD waveguides, J. Geophys. Res., 100, 79978002,1995

Guglielmi, A., J. Kangas, K. Mursula, T. Pikkarainen, O. Pokhotelov, and A. Potapov, Pc 1-induced electromagnetic lift of the background plasma in the magnetosphere, J. Geophys. Res., 101, 21493-21500, 1996.

Gustafsson, G., M. Andre, L. Matson, and H. Koskinen, On waves below the local proton gyrofrequency in auroral acceleration regions, J. Geophys. Res., 95, 5889-5904, 1990.

Hultqvist, B., On the acceleration of positive ions by high-latitude, largeamplitude electric field fluctuations, J. Geophys. Res., 101, 27111-27124, 1996.

Kangas, J., A. Guglielmi, and O. Pokhotelov, Morphology and physics of short-period magnetic pulsations (A Review), Space Sci. Rev., 83, 435$512,1998$.

LaBelle, J. and R. A. Treumann, Poynting vector measurements of electromagnetic ion cyclotron waves in the plasmasphere, J. Geophys. Res., 97 13789-13797, 1992

Landau, L. D. and E. M. Lifshitz, Electrodynamics of Continuous Media, 460 pp., Pergamon, Oxford, 1984.

Lundin, R., Acceleration/Heating of plasma on auroral field lines: Preliminary results from the Viking satellite, Ann. Geophys., 6, 143-152, 1988

Lundin, R. and B. Hultqvist, Ionospheric plasma escape by high-amplitude electric fields: Magnetic moment “pumping”, J. Geophys. Res., 94, 66656680, 1989

Lundin, R. and L. Eliasson, Auroral energization processes, Ann. Geophys., 9, 202-223, 1991

Lundin, R., G. Gustafsson, A. I. Eriksson, and G. Marklund, On the importance of high-latitude low-frequency electric fluctuations for the escape of ionospheric ions, J. Geophys. Res., 95, 5905-5919, 1990.

Miller, M. A., Motion of charged particles in the high-frequency electromagnetic fields, Radiophysics, 1, No. 3, 110-123, 1958 (in Russian).

Mursula, K., L. G. Blomberg, P.-A. Lindqvist, G. T. Marklund, T. Bräysy, R. Rasinkangas, and P. Tanskanen, Dispersive Pc 1 bursts observed by Freja, Geophys. Res. Letters, 21, 1851-1854, 1994.

Northrop, T. G., The Adiabatic Motion of Charged Particles, 109 pp., WileyInterscience, New York, 1963.

Perraut, S., R. Gendrin, A. Roux, and C. de Villedary, Ion cyclotron waves: direct comparison between ground-based measurements and observations in the source region, J. Geophys. Res., 89, 195-202, 1984.

Pitayevsky, L. P., Electric forces in a transparent dispersive medium, JETP , 39, No. 5(11), 1450-1458, 1960 (in Russian).

Singh, N., Ponderomotive versus mirror force in creation of the filamentary cavities in auroral plasma, Geophys. Res. Lett., 21, 257-260, 1994.

Washimi, H. and V. I. Karpman, On the ponderomotive force of a high frequency electromagnetic field in a dispersive medium, JETP, 71, 10101016, 1976 (in Russian)

A. Guglielmi (e-mail: gugl@uipe-ras.segis.ru), K. Hayashi (e-mail: hayashi@geoph.s.u-tokyo.ac.jp), R. Lundin (e-mail: rickard@irf.se), and A. Potapov (e-mail: potapov@iszf.irk.ru) 